\title{
THE ALLOMETRIC HYPOTHESIS WHEN THE SIZE VARIABLE IS UNCERTAIN: ISSUES IN THE STUDY OF CARCASS COMPOSITION BY SERIAL SLAUGHTER
}

\author{
A. B. PLEASANTS ${ }^{1}$, G. C. WAKE ${ }^{2}$ and A. L. RAE ${ }^{3}$
}

(Received 21 February 1995; revised 17 July 1995)

\begin{abstract}
The allometric hypothesis which relates the shape $(y)$ of biological organs to the size of the plant or animal $(x)$, as a function of the relative growth rates, is ubiquitous in biology. This concept has been especially useful in studies of carcass composition of farm animals, and is the basis for the definition of maintenance requirements in animal nutrition.

When the size variable is random the differential equation describing the relative growth rates of organs becomes a stochastic differential equation, with a solution different from that of the deterministic equation normally used to describe allometry. This is important in studies of carcass composition where animals are slaughtered in different sizes and ages, introducing variance between animals into the size variable.

This paper derives an equation that relates values of the shape variable to the expected values of the size variable at any point. This is the most easily interpreted relationship in many applications of the allometric hypothesis such as the study of the development of carcass composition in domestic animals by serial slaughter. The change in the estimates of the coefficients of the allometric equation found through the usual deterministc equation is demonstrated under additive and multiplicative errors. The inclusion of a factor based on the reciprocal of the size variable to the usual log - log regression equation is shown to produce unbiased estimates of the parameters when the errors can be assumed to be multiplicative.

The consequences of stochastic size variables in the study of carcass composition are discussed.
\end{abstract}

\section{Introduction}

The relationships between the structural components of plants and animals are constrained by their physical environment. For example, the legs of an animal must have

\footnotetext{
${ }^{1}$ Agresearch, Whatawhata research Centre, Private Bag, Hamilton, New Zealand

${ }^{2}$ Department of Mathematics, Massey University, Palmerston North, New Zealand

Correspondence address: Dept. Mathematics, The University of Auckland, Auckland, New Zealand

${ }^{3}$ Department of Animal Science, Massey University, Palmerston North, New Zealand

(C) Australian Mathematical Society, 1997, Serial-fee code 0334-2700/97
} 
a certain minimum bone diameter if they are to support the animal of a given weight. In effect this means that the relative proportions of the structural components must change as an organism grows if functionality is to be preserved. Other examples relate the surface area of animals to their volume, the relationship being constrained by the physical problems of heat loss. This is the principle of allometry formulated by Huxley [7], and elaborated upon by Gould [6], and Sprent [15].

The allometric hypothesis in its simplest form implies that the relative growth of an organ or a part is in constant proportion to the relative growth of the organism or the whole. That is,

$$
\frac{1}{y} \frac{d y}{d t}=\frac{k}{x} \frac{d x}{d t}
$$

where $k$ is the dimensionless constant of proportionality called the maturity.

The part variable $(y)$ is usually called the shape variable, and the whole variable $(x)$ is called the size variable. Allometry was first used to study the way the shape of organs changed as the size of the organism increased when the relationship between the relative growth rates described by equation (1) held.

Recently the allometric hypothesis has been used to study changes in the carcass composition of farm animals for the meat production (Murray and Slezacek [13]; Jury et al. [8]; Kirton et al. [10]). There are commercial advantages to the manipulation of carcass components to emphasise the production of lean meat and reduce the production of fat.

To assist this study, changes in carcass composition which result from nutritional or environmental effects need to be distinguished from changes due to differences in the relative growth rate of carcass components as the animal ages and grows larger. The allometric hypothesis describes a suitable model for changes in the relative weight of different carcass components as the size of animal increases. For example, if the weight of fat in carcass was of interest fat weight would increase in proportion to the increase in carcass weight according to the allometric relationship expressed in equation (1). That is, in terms of the original allometric formulation fat weight acts as a shape variable and carcass weight is the size variable.

Measurement of carcass components which deviate from the relationship between component weight and animal size expressed by the allometric hypothesis can be investigated for the influence of nutritional or environmental effects. This makes the allometric hypothesis an attractive basis for statistical models to study carcass composition.

The allometric relationship between carcass components and carcass size may not be constant over the life of the animal, but depend on the maturity of the animal (Butterfield et al. [3]). Bone matures early and fat tissue matures late. Thus Lohse et al. [12] showed that piecewise log - log regressions provided better fits for some 
tissues than a single $\log$ - log regression, since the allometric coefficients changed as the maturity of the tissue changed.

However carcass studies involving slaughter of the animal have measurements that can only be made at one point in time. So changes in shape due to changes in size are inferred on the basis of comparisons between different animals at different sizes (size allometry), rather than comparisons between the same animal at different sizes (growth allometry). Size allometry reqires consideration of between animal variance as well as within animal variance in the allometric hypothesis.

If the independent size variable $(x)$ is random then equation (1) is a stochastic differential equation. There are additional considerations in the solution of stochastic differential equations not present in the deterministic case.

The Ito and Stratonovich calculus considers the evolution at a random value of the independent variable. In this problem we address the issue of finding the expected value of $y$ at the expected value of $x$.

The problem is illustrated in Figure 1. Here it can be seen that equivalent fluctuations about a given value of the independent variable $x=x^{*}$ do not produce identical fluctuations about the value of the response variable $y=y^{*}$ mapped by $x^{*}$. That is in general $E[f(x)]$ does not equal $f(E[x])$. For example if $x$ is a normally distributed random variable, and $y=\ln (x)$, then $\ln (E[x])$ corresponds to the median of $y$, not the expected value of $y$.

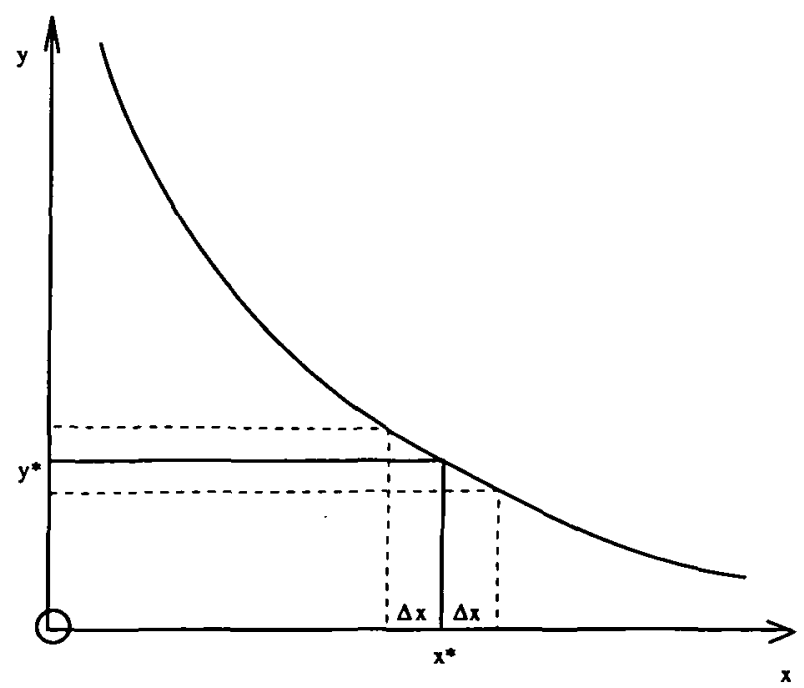

FIGURE 1. The asymmetry in the response ( $y$ ) to equal changes in the independent variable $(x) x+\Delta x$ $x-\Delta x$.

Stochastic differential equations may be handled using the Ito or Stratonovich 
calculus (Gard [5]). These methods consider the evolution of the state equation under random perturbations, and could be applied to the stochastic allometric equation we have defined. However, many biological applications are interpreted through estimates of the shape variable $(y)$ at the expected value of the size variable $(x)$. For example in studies of the evolution of carcass composition by serial slaughter animals slaughtered at different values of the size variable are randomly chosen to represent the mean of the population of interest. In this paper we address this problem of finding an equation for the evolution of shape $(y)$ as the expected value of the size variable $(x)$ changes while the allometric relationship defined by equation (1) holds.

When the relationship between the random variables is a differential equation the asymmetry in the response variable due to symmetric fluctuations in the independent variable (as in Figure 1) will lead to an expected evolution of the stochastic equation which is different to the evolution of the deterministic form. It is important to emphasise that the random elements of $x$ affect the evolution of the equation.

The goal of most serial slaughter experiments is to estimate the evolution of the response $(y)$ as the population mean of the size variable $(x)$ evolves through time. However, as noted the deterministic solution of equation (1) will not give this relationship when the size variable is random due to the symmetry of the response to random fluctuations in the size variable. Similar problems arise in many aspects of biology where the allometric hypothesis (and alternative formulations) have been used.

We emphasise that we are considering estimates of the parameters of the allometric equation as they are defined by the differential equation (1) when the size variable is random. In this case the uncertainty associated with the size variable $(x)$ interacts with the evolution of the equation in an asymmetric way as illustrated in Figure 1. Therefore the estimates of the parameters found by adding random error to the solution of the deterministic differential equation (1) are not the estimates of the parameters of equation when this equation is stochastic, that is, a different statistical model is involved.

This paper considers the estimation of the parameters of the allometric equation (1) when the size variable is random. We derive a solution of the stochastic allometric equation which gives the value of the part variable $(y)$ at the expected value of the size variable $(x)$.

\section{The stochastic allometric equation and its solution}

The independent variable time can be eliminated from the relative growth rate equation (1) to relate the change in the shape and size variables, that is,

$$
\frac{d y}{d x}=\frac{k y}{x} .
$$


This equation is easily solved by separation of variables to give

$$
y=C x^{k},
$$

where $C$ is the constant of integration. This expresses the well-known power law of allometry. In fitting this relationship to data it is usual to use the logarithm of both variables and fit the linear equation

$$
\ln y=\ln C+k \ln x,
$$

with appropriate errors at the data points $\left(x_{i}, y_{i}\right), i=1,2, \ldots, n$.

To derive the solution of equation (2) when the size variable is stochastic we proceed as follows.

We expand equation (2) in a series about the expected value $\mu$ of $x$. We write

$$
\mu=\int_{x \in R} x d F(x),
$$

where $R$ is a feasible region of the size variable.

The equation (2) becomes

$$
\frac{d y}{d x}=\frac{k y}{x}=k\left\{g(\mu)+(x-\mu) g^{\prime}(\mu)+\frac{(x-\mu)^{2}}{2} g^{\prime \prime}(\mu)+\ldots\right\},
$$

where

$$
g(x)=\frac{k y(x)}{x}
$$

So

$$
g^{\prime}(x)=\frac{k\left(x y^{\prime}(x)-y(x)\right)}{x^{2}}=k(k-1) \frac{y(x)}{x^{2}},
$$

using the original equation (2).

Then

$$
g^{\prime \prime}(x)=k(k-1) \frac{\left(x^{2} y^{\prime}(x)-2 x y(x)\right)}{x^{4}}=\frac{k(k-1)(k-2) y(x)}{x^{3}} .
$$

Substitution of this in the above, taking moments, and assuming that the moments higher than two are negligible, we obtain for the expected values on the right-hand side (RHS)

$$
E(\mathrm{RHS})=k y(\mu)\left\{\frac{1}{\mu}+\frac{\sigma^{2}(k-1)(k-2)}{2 \mu^{3}}\right\},
$$


and $\sigma^{2}$ is the variance of the size variable $x$.

We can evaluate the expected value on the left-hand side as follows:

$$
\begin{aligned}
E\left(\frac{d y}{d x}\right) & =\int_{R}\left(\frac{d y}{d x} d F(x)\right) \\
& =\int_{R} \lim _{h \rightarrow 0}\left(\frac{y(x+h)-y(x)}{h}\right) d F(x) \\
& =\lim _{h \rightarrow 0} \frac{1}{h}(E(y(x+h)-y(x))) \\
& =\lim _{h \rightarrow 0} \frac{1}{h} E\left(y(\mu)+(x+h-\mu) y^{\prime}(\mu)+\ldots-y(\mu)-(x-\mu) y^{\prime}(\mu) \ldots\right),
\end{aligned}
$$

and so

$$
E\left(\frac{d y}{d x}\right)=\frac{d y}{d \mu}
$$

where we have neglected the next term (which involves $d^{3} y / d \mu^{3}$ ) and those that follow. Using this in equation (6) we obtain the modified allometric differential equation

$$
\frac{d y}{d \mu}=k y(\mu)\left\{\frac{1}{\mu}+\frac{\sigma^{2}(k-1)(k-2)}{2 \mu^{3}}\right\}
$$

with solution (obtained again by separation of variables)

$$
y(\mu)=c \mu^{k} \exp \left(\frac{-k(k-1)(k-2) \sigma^{2}}{4 \mu^{2}}\right) .
$$

It is clear from equation (8) that the degree of bias associated with treating the differential equation (2) as deterministic, when however the size variable is random, depends on the size of the maturity coefficient $k$, and the square of the coefficient of variation $(\sigma / \mu)$ of the size variable. When the coefficient of variation is small then there will be little bias and the deterministic allometric equation will usually be an adequate description. However, as noted, when size allometry is used, the variance between animals will increase the coefficient of variation, and the bias in the estimation may be substantial.

\section{Estimates of the parameters in the allometric equation from data}

We will consider the least squares estimation of the parameters in the allometric equation. These are the coefficients of the maturity $(k)$ and the constant of proportionality (c), from data. 
3.1. Additive Errors The statistical model for the allometric hypothesis under additive errors when the size variable is not stochastic is

$$
y_{i}=c x_{i}^{k}+e_{i}
$$

and when the size variable is stochastic

$$
y_{i}=c x_{i}^{k} \exp \left[-k(k-1)(k-2) \sigma^{2} / 4 x_{i}^{2}\right]+e_{i} .
$$

The estimate of $c$ form equation (10) will be biased by amount $\exp [-k(k-1)(k-$ 2) $\left.\sigma^{2} / 4 x_{i}^{2}\right]$.

To estimate the maturity coefficient $k$ by least squares when the size variable is not stochastic we have

$$
c \sum_{i} x_{i}^{2 k} \ln x_{i}=\sum_{i} y_{i} x_{i}^{k} \ln x_{i}
$$

When the size variable is stochastic we get

$$
q=\sigma^{2} / 4 x_{i}^{2},
$$

with

$$
\begin{aligned}
\frac{\partial \sum e_{i}^{2}}{\partial k}= & -c^{2}\left(3 k^{2}-6 k+2\right) q e^{-k(k-1)(k-2) q} \sum_{i} y_{i} x_{i}^{k}-c e^{-k(k-1)(k-2) q} \sum_{i} y_{i} x_{i}^{k} \ln x_{i} \\
& +c^{2}\left(3 k^{2}-6 k+2\right) q e^{-2 k(k-1)(k-2) q} \sum_{i} x_{i}^{2 k}+c e^{-2 k(k-1)(k-2) q} \sum_{i} x_{i}^{2 k} \ln x_{i} \\
= & 0 \quad \text { for a minimum. }
\end{aligned}
$$

Hence

$$
\begin{aligned}
c e^{-k(k-1)(k-2) q}[ & c\left(3 k^{2}-6 k+2\right) q \sum_{i} x_{i}^{2 k}+c \sum_{i} x_{i}^{2 k} \ln x_{i} \\
& \left.-\left(3 k^{2}-6 k+2\right) q \sum_{i} y_{i} x_{i}^{k}-\sum_{i} y_{i} x_{i}^{k} \ln x_{i}\right]=0 .
\end{aligned}
$$

To show the estimates of $k$ from equation (9) equal the estimates of $k$ from this equation we need to show that the expression

$$
\begin{array}{ll}
c\left(3 k^{2}-6 k+2\right) q \sum_{i} x_{i}^{2 k}-\left(3 k^{2}-6 k+2\right) q \sum_{i} y_{i} x_{i}^{k}=0 \\
\Leftrightarrow & c \sum_{i} x_{i}^{2 k}-\sum_{i} y_{i} x_{i}^{k}=0 \\
\Leftrightarrow & \frac{\sum_{i} y_{i} x_{i}^{k}}{\sum_{i} x_{i}^{2 k}} \sum_{i} x_{i}^{2 k}-\sum_{i} y_{i} x_{i}^{k}=0 .
\end{array}
$$


This last statement is clearly true.

Hence $k=k^{\prime}$ and the estimate of the maturity $k$, ignoring the stochastic nature of the size variable will be unbiased.

3.2. Multiplicative errors Estimation of the parameters of the allometric equation (3) when the errors are assumed to be multiplicative, is usually carried out by taking logs of the shape and size variables, then estimating the coefficient by ordinary linear regression.

Thus

$$
\ln y_{i}=\ln c+k \ln x_{i}-k(k-1)(k-2) q+e_{i}
$$

and

$$
\frac{\partial \sum e_{i}^{2}}{\partial c}=2 \sum_{i}\left[\ln y_{i}-\ln a c-k \ln x_{i}+k(k-1)(k-2) q\right]\left(\frac{1}{c}\right)=0
$$

for a minimum.

Hence

$$
\begin{aligned}
\ln c & =\overline{\ln y_{i}}-k \overline{\ln y_{i}}+\sum_{i} k(k-1)(k-2) q / n, \\
c & =\frac{\dot{y}}{\dot{x}^{k}} \exp (k(k-1)(k-2) q),
\end{aligned}
$$

and so

$$
\hat{c}=\hat{c}^{\prime} \exp (k(k-1)(k-2) q),
$$

where $\dot{y}, \dot{x}$ are the geometric means of $y_{i}$ and $x_{i}(i=1, \ldots, n)$ respectively.

Thus the bias in the coefficient of proportionality $(c)$ is the same under both multiplicative and additive models.

The least squares estimate of the maturity $k$ under the multiplicative model remains biased by an amount

$$
k(k-1)(k-2) q \sum_{i} \ln x_{i}
$$

Clearly fitting the traditional allometric equation (7) will give unbiased estimates of $c$ and $k$ whenever $k$ equals zero, one or two.

3.3. Obtaining unbiased estimates for the stochastic allometric equation Unbiased estimates of the integration constant $c$ and the maturity $k$ can be found by 
fitting equation (10) in the case of additive errors, or for multiplicative errors the equation

$$
\ln y_{i}=\ln c+k \ln x_{i}-b / x_{i}^{2}+e_{i},
$$

where $b=k(k-1)(k-2) \sigma^{2}$.

Difficulties in estimation may result if the range of $x$ is restricted causing a high correlation between $\ln x_{i}$ and $1 /\left(x_{i}^{2}\right)$. In this case principle components regression may be employed to obtain stable regression coefficients. Transformation of the principle component regression coefficients back to the original scale will give unbiased estimates of $c$ and $k$ (Wonnacott and Wonnacott [17]).

TABLE 1. Simulated values for weight of fat $(\mathrm{kg})$ and corresponding weight of carcass $(\mathrm{kg})$ for an allometric relationship based on a constant of proportionality $a=0.05$ and maturity $k=1.5$.

\begin{tabular}{|c|c|c|c|}
\hline $\begin{array}{c}\mathbf{y} \\
\text { fat wt }\end{array}$ & $\begin{array}{c}\mathbf{x} \\
\text { carcass wt }\end{array}$ & $\begin{array}{c}\mathbf{y} \\
\text { fat wt }\end{array}$ & $\begin{array}{c}\mathbf{x} \\
\text { carcass wt }\end{array}$ \\
\hline 0.7616 & 6.3939 & 1.8783 & 11.2407 \\
0.8164 & 5.1591 & 1.9211 & 12.5111 \\
0.8366 & 7.4612 & 1.9732 & 10.6565 \\
0.8849 & 6.1437 & 2.0231 & 12.8770 \\
0.9312 & 6.2790 & 2.0697 & 14.1175 \\
0.9700 & 6.4997 & 2.1358 & 13.2353 \\
0.9997 & 6.4705 & 2.1782 & 12.2180 \\
1.0318 & 7.1284 & 2.2479 & 13.4181 \\
1.1025 & 6.7268 & 2.2919 & 12.9622 \\
1.1570 & 8.1245 & 2.3358 & 13.6628 \\
1.1665 & 9.2445 & 2.3995 & 14.2555 \\
1.2335 & 9.9586 & 2.4505 & 13.6355 \\
1.2605 & 7.9496 & 2.5031 & 14.3318 \\
1.2998 & 7.7626 & 2.5611 & 14.7429 \\
1.3535 & 10.8612 & 2.6316 & 11.5878 \\
1.4005 & 9.9600 & 2.6592 & 11.6541 \\
1.4255 & 11.0240 & 2.7513 & 11.1668 \\
1.4875 & 9.7317 & 2.7840 & 13.3119 \\
1.5277 & 11.5991 & 2.8561 & 13.6626 \\
1.5753 & 11.1954 & 2.9053 & 13.2678 \\
1.6323 & 9.2611 & 2.9566 & 16.4991 \\
1.6701 & 10.3982 & 3.0102 & 16.1972 \\
1.7253 & 10.1323 & 3.0856 & 17.7749 \\
1.7815 & 10.4528 & 3.1415 & 14.2400 \\
1.8242 & 12.2392 & 3.2031 & 16.8683 \\
\hline
\end{tabular}


Example The data in Table 1 are simulations of fat and carcass weight measurements of lambs from an investigation by Butler-Hogg [2]. The simulation was for an allometric relationship between fat and carcass weight with integration constant $c=0.05 \mathrm{~kg}$ and maturity $k=1.5$. Carcass weights were simulated from a normal distribution with a standard derivation of $1.5 \mathrm{~kg}$, thought to model the variance associated with size allometry. The error structure was additive. Using least squares to estimate the coefficients of equation (3) using log - log regression yields estimates of $c=0.09$ and $k=1.27$. Failure to account for the random nature of the size variable has biased the estimate of $c$ by $60 \%$. The bias in the parameter $k$ is mild.

Fitting the regression equation (9) produced estimates of $c=0.04$ and $k=1.52$, very close to the figures used to construct the simulation, suggesting that unbiased estimates of the parameters of the allometric equation when the size variable is random can indeed be obtained from equation (9).

\section{Discussion}

The relationship between the shape variable $(y)$ and the expected value of the size variable $(x)$ in the allometric equation (1) is different from the deterministic case when the size variable is uncertain. The difference depends on the magnitude of the maturity $(k)$ and the magnitude of the coefficient of variation $\left(\sigma^{2} / 4 \hat{\mu}^{2}\right)$ of the size variable. However unbiased estimates of parameters can be found by fitting equation (10) when errors are additive, or equation (12) if errors are multiplicative.

In many studies the allometric equation is used as an empirical model to test statistical hypotheses, that is, the log-log model is fitted to data. Interpretation may not depend on the allometric hypothesis as defined by equation (1). In such circumstances there is no problem with this procedure. However, if the interpretation of the analysis does depend on the relationship expressed in equation (1) and (2) the issues raised in this paper should be considered. For example, biological inferences may be drawn from estimates of maturity $(k)$ which are less than or greater than 1 . This infers that the part is changing slower than, or faster than, the size. Incorrect conclusions may follow if the wrong model is used to settle such a question.

The allometric principle has become the basis for describing changes in carcass composition (fat, bone, lean), as carcass weight increases (Butler-Hogg [2]; Seebeck [14]; Korver et al. [11] and Blaxter [1]). When this description is extended to computer models then the predictive value of allometry becomes important. In this respect the changes of the allometric state equation due to a random size variable will have an influence, especially if extrapolation occurs. For example, a coefficient of variation of $30 \%$ in the size variable will result in the fat content of the carcass being underestimated by $10 \%$. When parameters are determined on the basis of the 
size allometry, and predictions are made from the mean of the data estimating the coefficient, then the errors of prediction will be large and bias in the model predictions will increase.

The allometric hypothesis, or its derivatives are the basis for carcass composition models discussed by Emmans [4], Keele et al. [9] and Williams et al. [16]. This work shows that a correction needs to be made to account for the stochastic nature of the size variables if predictions from such models are to be accurate, especially at the extremes. Derivatives of the allometric hypothesis such as the model discussed by Emmans [4] need to be examined to find the consequences of stochastic size vairables on the predictions that they make.

When an estimate of the variance of the size variable is not available, including an independent variable in the regression based on the reciprocal of the square of the size variable will allow unbiased estimation of the parameters of the allometric equation. However care should be taken because problems of multicollinearity may arise if the range of the size variable in an experiment is small. Use of principle component regression may be advised if this occurs.

The allometric hypothesis is a valuable concept for studies of carcass composition in farm animals. This paper has developed modifications to the allometric state equation when the size variable is random. This should assist the accuracy of theories of carcass composition based on the allometric hypothesis.

\section{Acknowledgement}

The advice of Professor G.A. Seber, University of Auckland, concerning the derivation in Section 2 is gratefully acknowledged.

\section{References}

[1] K. L. Blaxter, V. R. Fowler and J. C. Gill, "A study of the growth of sheep to maturity", J. Agric. Sci. 98 (1982) 405-420.

[2] B. W. Butler-Hogg, "The growth of Clun and Southdown sheep: Body composition and the partitioning of total body fat", Animal Production 39 (1984) 405-411.

[3] R. M. Butterfield, D. A. Griffiths, J. M. Thompson, J. Zamora and A. M. James, "Changes in the body composition relative to weight and maturity in large and small strains of Australian merino rams", Animal Production 36 (1983) 29-37.

[4] G. C. Emmans, "Genetic components of potential and actual growth", Brit. Soc. of Animal Production Occasional Publications (1988) 153-181.

[5] T. C. Gard, Introduction to stochastic differential equations (Marcel Dekker, New York and Basel, 1988).

[6] S. J. Gould, “Allometry and size in ontogeny and phylogeny", Biol. Rev. 41 (1966) 587-640. 
[7] J. S. Huxley, Problems of relative growth (Methuen, London, 1932).

[8] K. E. Jury, P. D. Fourie and A. H. Kirton, "Growth and development of sheep. 4. Growth of musculature", N.Z. J. Agric. Res. 20 (1977) 115-121.

[9] J. W. Keele, C. B. Williams and G. L. Bennet, "A computer model to predict the effects of level of nutrition on composition of empty body gain in beef cattle: 1 . Theory and development", $J$. Animal Sci. 70 (1992) 941-957.

[10] A. H. Kirton, D. C. Dalton, G. Winn and D. M. Duganzich, "Body composition of cull Romney, Dorset $x$ Romney, and Cheviot ewes from New Zealand hill country", N.Z. J. Agric. Res. 28 (1985) 241-247.

[11] S. Korver, M. W. Tess, T. Johnson and B. B. Anderson, "Size-scaled lean and fat growth patterns of serially slaughtered beef animals", J. Animal Sci. 64 (1987) 1292-1301.

[12] C. L. Lohse, F. P. Moss and R. M. Butterfield, "Growth patterns of muscles of merino sheep from birth to 517 days", Animal Production 13 (1971) 117-126.

[13] D. M. Murray and O. Slezacek, "The effect of growth rate on muscle distribution in sheep", $J$. Agric. Sci. 85 (1975) 189-191.

[14] R. M. Seebeck, "Factors affecting patterns of development and their assessment", Animal Production 37 (1983) 53-65.

[15] P. Sprent, "The mathematics of size and shape", Biometrics 28 (1972) 23-41.

[16] C. B. Williams, J. W. Keele and G. L. Bennett, "A computer model to predict the effects of level of nutrition on composition of empty body gain beef cattle: 2 . Evaluation of the model", J. Animal Sci. 70 (1992) 858-866.

[17] R. J. Wonnacott and T. H. Wonnacott, Econometrics, second ed. (Wiley, New York, 1979). 